\title{
SEMANTIC ANALOGY*
}

(Received 19 November, 1979)

\begin{abstract}
Even a three-year-old child can understand a story about 'people too little to see'... Hilary Putnam, 'What Theories Are Not'

If one has to imagine some one else's pain on the model of one's own, this is none too easy a thing to do: for I have to imagine pain I do not feel on the model of the pain which $I$ do feel. - Wittgenstein, Phil. Investigations
\end{abstract}

Faced with the difficulty of rationalizing the intuitive non-deductive principles of inference we employ as guides to a transcendent truth one may be tempted, in the vein of Peirce, to offer a reductive account of what truth is, taking it to be that which is, if only in the 'long run', that which is warrantedly assertible.

Similarly, faced with the apparently intractable problems one encounters when one takes meaning, to be characterized in terms of truth-conditions, there is a strong temptation to offer an account of sense which ties it primarily to the conditions under which we are warranted in asserting and denying a proposition. Such a verificationist account of meaning is supported even more strongly by the doctrine of meaning as use, and by arguments to the effect that learning the meaning of an expression can only be learning when it is appropriate to use it or to accept or criticize another's use of it; and, hence, that what is learned when meaning is grasped must be, primarily, these very conditions of acceptancy and rejection.

That an adequate theory of meaning will invoke the conditions under which we are warranted in accepting and rejecting an assertion at some points in its structure is something many will accept. But a very fundamental question is this: Must an account of the meaning of an assertion be an account of 
the grounds for accepting and rejecting it for every assertion whose meaning is to be characterized? Or might the situation be, rather, that for some assertions one must, to give their meaning, stipulate their verification and falsification conditions, but this need not be done assertion-by-assertion for every proposition accepted as meaningful? Could we not, in some way, project an understanding of meaning throughout language, using its structural features as the guide to correct projection, from some fundamental basis to which meaning accrues in a manner acceptable to a verificationist?

The basic idea is simple, if, admittedly, terribly vague. For those propositions whose truth-conditions are accessible to us, meaning is given by stipulating the conditions of warranted acceptability and rejectability. For these propositions giving the verification and falsification conditions is giving the truth-conditions and, surely, on anyone's view of meaning, fully characterizing the meaning. But for those propositions whose truth-conditions are, somehow, 'inaccessible' to us we are blocked from this route. The solution is not to force us to reject our intuitive picture of the proposition as having inacessible truth-conditions, offering a verificationist revision of just what the meaning of the proposition 'really' is; but, rather, to offer an account of how we can grasp the meaning of these propositions without stipulating for them directly conditions of warranted assertability and rejectability. Instead we offer an account of how an understanding of these propositions arises indirectly out of their structural relationship, intralinguistically, to the propositions whose truth conditions are open to us.

It is one version of such a theory that I intend to look at here.

It would be useful here to make some brief remarks about the overall structure of various aspects of the traditional verificationist program. While everyone is familiar with many of the objections in detail which apparently vitiated these programs, some remarks about the general structure of the alleged failures will be important here.

The positivists tried to present both criteria of meaningfulness and a theory of meaning. The criteria of meaningfulness were usually framed in terms of sentences, principles being offered to determine when a proposition was meaningful in terms of its bearing some appropriate logical relationship to the distinguished class of observational, and hence prima facie meaning- 
ful, propositions. Of course there is also a tradition of attempting to apply a criterion of meaningfulness for terms as well. Theories of meaning were presented in the form of a theory of how terms accrue meaning on the basis of an antecedently given observational vocabulary. That a theory of meaning would be given as a theory of term meaning seems an inevitable consequence of componentialism, i.e. of the realization that a theory of meaning must proceed by terms unless an infinite number of distinct sentences are to have meaning assigned to them one at a time. Curiously not much attention was paid by the verificationists to the fact that even if we could characterize the class of meaningful terms, and specify their meanings, we would still need a theory which tells us when a given combination of terms into a sentence is meaningful and which tells us how to generate the meaning of the whole sentence out of the meanings of the component terms. This will be essential for us later.

The history of the verificationist programs is a history of progressive weakening. First only sentences strictly verifiable and strictly falsifiable are considered meaningful. But then vast numbers of prima facie meaningful propositions of science are declared metaphysical or, at least, unscientific. So the criterion is weakened in one direction or another. Well known is the direction in which one allows the use of auxiliary sentences in testing a sentence for meaningfulness by exploring its deductive connections with observational sentences. But then it became hard to formulate a criterion which excludes any sentence at all from the realm of the meaningful. Alternatively, one can weaken the criterion by allowing inductive or probabilistic relations between observation sentence and theoretical sentence rather than insisting on a strict deductive relation between the two. Or one could move in both directions simultaneously. If one chooses to deal with terms rather than sentences, a parallel process of weakening is observed as the authors move from operational or coordinative definitions, to reduction sentences, to the holistic notion of partial interpretation. Here again, by allowing inductive connections between observational consequences and theory to legitimize a theory, and hence to give meaning to the terms appropriately appearing in it, a weakening in the other direction is invoked as well.

It is well known that the weakening of the original strict verificationist criteria cannot be accomplished without severe difficulties infecting the program. Once allow as meaningful an assertion whose truth-conditions cannot be fully stipulated in terms of the observational vocabulary and it 
becomes hard to see how any sentence or term can be excluded from the realm of the meaningful. This is most obvious when the move toward holism is made, that is when 'auxiliary sentences' are allowed in testing a sentence for meaningfulness or when 'partial interpretation' of terms replaces strict operational definitions. What we must be clear about, though, is that the failure to produce a definitive verificationist criterion of meaningfulness is not the result of mere technical difficulties, but is, instead, the result of very serious difficulties the approach faces as soon as it abandons the most naive and rigid standards of strict necessary and sufficient truth conditions framed in the observation language.

Each direction of weakening, the holistic which allows the invocation of auxiliary sentences when testing a sentence for meaningfulness or the invocation of other theoretical terms when testing a term for meaningfulness, and the criterial which allows the use of inductivist or probabilistic connections between the theoretical and the observational to serve as the test for meaningfulness, suffers its own characteristic difficulties. The real force of these difficulties only becomes apparent when one moves from the comparatively easy task of specifying a criterion for meaningfulness to the much more difficult task of offering a full-fledged theory of meaning for theoretical discourse.

The holistic case is the more familiar. Once take whole theories as the appropriate bearers of meaning, and, in the domain of inference, take responsibility to the phenomena as the basic criterion of warrant for their acceptability (allowing, possibly, as well simplicity, methodological conservatism, et al. to also play a role), it then becomes hard to see how either independent non-theory-relative meaning is to be assigned to any sentential atom of the theory, or independent reference and extension to that sentence's component singular terms and predicates. On the inferential plane it is hard to see how independent warrant is to be assigned to sentences one at a time. This difficulty shows itself most clearly in the familiar feature of duality. Given a theory which adequately saves the phenomena we can also cook up innumerable others, superficially inconsistent with the given original theory, by simply .compensating for variation in one place with a saving variation elsewhere. On the level of inference such duality leads to skepticism, a priorism or, their not-particularly-intelligible relative, conventionalism. But, much deeper, is what duality leads to one the level of meaning theory. Either directly, as in Eddington, Reichenbach, et al., or more obscurely on the part 
of some recent authors, duality on the theoretical level leads us by a smooth chain of argument to a denial of genuine (or, perhaps, unrelativized) meaning (and reference) to the sentences of the theory taken one at a time or to their components. Truth is denied to the sentences individually, but said to be applicable to them only relative to the remaining body of theory; and the reference of the terms and predicates of the sentences is again now only 'relative' to the remaining theoretical context. Commonly (and plausibly) we are led by such arguments to the dilemma of either retreating to the reductionist analysis of the meaning of theoretical assertions which the strict verificationist had in mind in the first place, or, instead, to a denial of reductionism which succeeds in finding a role for the individual theoretical sentences by, in its meaning theory, denying them meaning properly so called at all - that is, by a retreat to instrumentalism for the theoretical apparatus.

The loosening of strict verificationism which proceeds by way of replacing entailment relations with relations of warranted assertability, inductive probability, etc. also faces serious problems. Here the fundamental difficulty is not of holism. In the case where verificationism was weakened by allowing in auxiliary sentences in tests of meaningfulness and by taking meaning as role played in total theory, the difficulty is in reconciling an empiricism which fundamentally takes whole theories as the units of meaning with an attempt to preserve notions of truth, reference, etc. distributed 'atomistically' over individual sentences and their component referring expressions. In this new case the problem is trying to reconcile the new theory of meaning appropriate to this weakened criterion of meaningfulness with the basic intuitions we have connecting the notions of meaning and truth.

It is almost a truism to claim that a stipulation of the meaning of an assertion requires at least a stipulation of its truth conditions. This might not be enough, as we know, because of all the familiar arguments to the effect that logically equivalent assertions need not be synonymous, etc. But, surely, one argues, saying under what circumstances an assertion is true and under what false constitutes a necessary component in specifying its meaning. Then one goes on to account for the meanings of the components of the sentence in terms of their contribution to the sense, i.e. the truth-condition stipulation, of the whole.

But if we are to be allowed to introduce novel assertions into our language on the basis solely of their inferential relations to already understood assertions, where these inferential relations are less then deductive and so are not 
sufficient even to characterize the truth-condition of the assertion so introduced, then what are we to make of this intuitively obvious claim that the meaning of an assertion is a stipulation of the conditions under which it is true and under which it is false?

The theory which takes the fundamental primitive in meaning theory to be warranted assertability rather than truth faces a dilemma. Either it reserves a place for a limited class of assertions whose meaning is given by our recognition of the truth-conditions for them (observation sentences, perhaps) or it does not. In the former case those assertions not in the privileged class seem to have such an attenuated sense of meaning left over for them (in comparison with that rich, full sense of meaning possessed by the members of the privileged class whose meaning is their recognizable truth-condition) that we are easily led to an instrumentalistic account of their role in our conceptual scheme. Their meaning doesn't refer to their truth-conditions, their singular terms don't have reference in the full sense, nor their predicates extensions. Why then take their apparent ontological commitments, etc. seriously at all?

The alternative is to hold that the meaning of all assertions is given by their conditions of warranted assertability and deniability and that the recognizability of truth-conditions never plays a role in the comprehension of meanings. Such a flat-out anti-truth-condition theory of meaning is hard to criticize, expecially since, despite Dummett's suggestive work, no such theory has even really been made available to us for our inspection. Dummett suggests to us that the adoption of all-out verificationism will, at the very least, require a revision in our logic, from traditional to intuitionistic logic or some variant thereof. Far more serious is the full anti-realism which one will then seem to be committed to. If all sentences have as their meaning something specified in terms of conditions of warranted assertability and deniability; if for all of them meaning is disconnected to the notion of truthcondition, and, hence, for their components, meaning is disconnected from the orthodox notions of reference and extension, what will be left of the connection between language and the world at all? It is one thing for Wittgenstein, advocating a verificationist account of mentalistic language, to tell us that a nothing would do as well as a something so far as the meaning of 'pain' is concerned. It is something else again to adopt such a cavalier attitude toward being in general.

The parallel here with the case of inference is clear. Once adopt a founda- 
tion language of propositions warranted by direct access to the states of the world they describe, and skepticism about the truth of the remaining sentences, believed on such far more slender warrant, rears its head. Deny the existence of foundation statements altogether and one quickly finds oneself on the slide from a coherence theory of warrant toward a coherence theory of truth. What we see here is that on the level of the theory of meaning, and even without the coherentist's holism, a doctrine which eliminates truth-conditions altogether in favor of conditions of warranted assent and dissent soon loses any ability to account for the connection between language and an independent world of things, properties and facts. Perhaps, once again, the world is well lost as Rorty would have us believe, but it is a radical step to take and one we would do well to hesitate before taking. Incidentally, this dilemma of either reserving a special class of observation sentences or else treating all sentences on a par gives rise to similar difficulties for the holist as well.

III

Given the difficulties of sustaining a realistic interpretation of terms and sentences referring to unobservables on either the holistic or verificationistic accounts, it would seem prudent to at least explore any prima facie viable alternative which presents itself. One such alternative has particular appeal since the model of understanding it presents is one which seems to us pretheoretically (rightly or wrongly) to have plausibility to it. The view is this: Basic sentences describing epistemically accessible states of affairs are learned by us by the presentation to us of the appropriate states of affairs. The meaning of the assertion is then given by association of it with its directly available truth-conditions. But such basic sentences are made up out of components and, indeed, on the model of any adequate theory of language based on a componentialist semantics (And what other kind could possibly explain our mastery of a potentially infinite collection of novel sentences?) the meaning of the whole sentence must be understood as being generated out of the meanings of the components and their grammatical assemblage into the whole sentence. But then we can understand, once again on a componentialist theory of meaning for the wholes as generated out of the meaning of parts, novel sentences constructed out of these very same elements, the elements first introduced to us through their role in sentences whose truth-conditions are open to our direct inspection. But these novel sentences may describe 
states of affairs not open to our observational capacities. Hence, on a basically verificationist theory of meaning, one can understand how it is that one can have an understanding of sentences which describe states of affairs whose truth-conditions cannot be 'presented' to us. But, on this account, the meaning of sentences is supposed to be given individually, rescuing us from the dualities of holism and its conventionalist consequences, and the meaning of the novel assertion is connected to its truth-condition, and not to conditions of warranted assertability and deniability, and, hence, the new approach is supposed to give us genuine reference for the components of the novel assertion and genuine truth for the assertion as a whole.

That such an account is fraught with difficulty is, I think, obvious. But that it is one many people intuitively accept on a pre-theoretical level is also clear. Knowing what 'red' means from such cases as 'This is a red apple' I have no difficulty in understanding what it is for something to be a red object too small to see. Knowing what pain is from my own case I understand (pace Wittgenstein) what it is for you to be in pain. Remembering the famous (notorious?) argument from analogy to the legitimacy of belief in your mental states on the analogy with my own, it becomes not implausible to call such a theory as the present one a theory of meaning by analogy. The general structure of the theory here looks like this: Initially meaning accrues to whole sentences by an association of the sentence with its (conventionally determined) truth-condition, which 'state of affairs' is directly open to epistemic access. Meaning then accrues to sentences in the language whose truth-conditions are not so accessible by a process of decomposition and reassembly which proceeds by an understanding of the way in which the meaning of whole sentences is formed out of the meaning of their parts.

I don't think that even that loose characterization will do for all those cases which one might call projection of meaning by analogy. For example, it is sometimes claimed that quantification over an infinite domain is understood 'analogically' from quantification over finite domains. Here the 'projection' of meaning by analogy does not seem obviously assimilable to the notion of sentential decomposition and reassembly.

As a first step at exploring the possibility of such a notion of 'semantic analogy' playing a respectable role in an overall semantic account of meaning, let us first look at a number of objections to such a theory of meaning accrual. These objections are basically of two kinds: from cases and from general principles. 
The refutation of semantic analogy by cases goes like this: The existence of obviously nonsensical sentences made up 'grammatically' out of parts which are plainly meaningful when they appear in other sentences shows us that projection of meaning by decomposition and reassembly is illegitimate. There are two kinds of cases, those of 'ordinary language': 'It is five o'clock on the sun'; and those of science: 'Two spatially separated events are simultaneous', 'This electron is green'.

Now of course we could deny the meaninglessness of the sentences adduced. But even if we accept them as meaningless, their existence doesn't show that projection of meaning by decomposition and reassembly is always illegitimate. It does show that it sometimes is. The existence of such cases (very familiar from Wittgenstein who wishes to break the alleged stranglehold of the 'false picture' of meaning by analogy has on us) does indeed show us that the advocate of the legitimacy of meaning by analogy has his work cut out for him. For he must make at least the first steps toward a theory of semantic projection which will delineate the range of legitimate projection of meaning by analogy. Such a theory might at least initially proceed by a theory of 'semantic categorization' of terms, marking out by means of construction rules legitimate from illegitimate combinations in terms of the semantic categories into which terms are placed. Such a theory would not, I believe, work in general. In any case, it could be but the most rudimentary intermediate step in our theory, for the very notion of semantic categorization itself calls out for explanation.

Here, as elsewhere, the parallel of the theory of meaning with the theory of believability is interesting. As we now know, the believer in inductive inference has his work cut out for him as well. Even prior to the establishment of a final 'justification' for inductive reasoning, he must first give us at least a rudimentary description of what inductive reasoning consists in. Even on the very naive model of inductive generalization as induction by enumeration, the existence of unnatural classifications of things (with 'grue' as the extreme case of an illegitimate classificatory term) shows that there is a fundamental problem first to be faced in trying to delineate the legitimate from the illegitimate cases of inference and to somehow rationalize (or at least explain) the boundary between the two.

Over and above the 'refutation by cases' (the form of refutation which appears very clearly in the relvant portion of Wittgenstein's Investigations) one can find attempts at more 'theoretical' refutations of the view that 
meaning can accrue by the analogy of epistemically unaccessible to epistemically accessible states of affairs.

One such refutation is that of Dummett. ${ }^{1}$ Basically, the line of argument is this: The theorist who believes that meaning can accrue by analogy is basically a 'realist' in semantics. That is, he takes the meaning of an assertion to be its truth-conditions. But then how do we understand the meaning of an assertion? Only by 'grasping' its truth-conditions. What can this amount to? Either being able to stipulate those truth-conditions in some non-trivial verbal way (say by offering a reductive analysis of the meaning of the assertion) or by knowing what it takes to determine the truth or falsehood of the assertion. Now consider those assertions whose truth-conditions outrun the limits of epistemic accessibility. The 'meaning by analogy' theorist envisages someone who can determine the truth or falsity of such an assertion by means 'analogous' but not identical to those we employ. It is such an extended notion of verification, he claims, which is sufficient to give meaning to these assertions, and reference to such an extended method of verification which, indeed, specifies what the meaning of these, in actual fact, unverifiable and unfalsifiable, assertions is.

Now, Dummett continues, where the analogy of the new extended method of verification to our usual methods is weak or non-existent, the analogist admits that meaning cannot accrue in this way. Hence, while it might be legitimate to think of meaning accruing to quantifications over infinite totalities by envisaging someone who can 'survey' an infinite class as we survey a finite class by exhaustive enumeration of cases, none but the most ardent analogist is likely to be satisfied with a theory of the meaning of modal assertions which gives us a verifier with the extended capability of surveying the contents of other possible worlds.

But, Dummett says, these distinctions are only of psychological importance, explanatory only of why the analogist thinks that he has a theory of meaning even in his best cases where the analogy is clearest. For even in those cases he is viewing the meaning of an assertion not as we use it but as his super-powered verifier uses it and, hence, as far as a theory of meaning of assertions in our language does, he is mis-representing the use of the assertion in language and mis-construing its meaning.

I think, though, that this refutation of what Dummett calls (curiously) 'strong realism' isn't conclusive against the analogist's case. Even if we take the analogy to be, as Dummett suggests, one between our actual methods of 
verification and those of an imagined super-being, is it so clear that the analogist is blocked in claiming that the presentation of such an analogy is enough to give us a grasp of the meaning of the assertion in question? The claim that this must be a misconstrual of the meaning as the method of verification described is not that actually employed by us is persuasive only if one has already accepted the verificationist's identification of the meaning of an assertion with its method of verification as it is actually used, which is, of course, exactly what the analogist is anxious to deny.

Furthermore, is it so clear that it is an analogy of methods of verification that the analogist really has in mind? To be sure he identifies the meaning of an assertion with its truth-conditions and grasping the meaning with 'grasping' the truth-conditions. But why should he agree that this can only amount to grasping that method by which we, or at least some analogically more powerful extension of our selves, would use to determine the truth or falsehood of the assertion? Once again that is only plausible if one is a verificationist to begin with. Most likely the analogist's position is, rather, something like this: For the basic assertions, those whose truth-conditions are directly accessible to us epistemically, we learn their meaning by associating them directly with the presented truth-condition. Of course since directly being aware of the existence of the truth-condition is the primary and most conclusive warrant we could possibly have for believing in these propositions, one could also identify the meaning of the assertion with the primary grounds for warranting belief in it - without committing oneself, of course, to a verificationist theory of meaning. For those assertions whose truth-conditions are not open to our epistemic access, we understand their meaning by grasping their truth-condition. We do this latter by seeing the analogy of the truthcondition of this new assertion with the truth-condition of some one or more of the basis assertions. But it is an analogy of truth-conditions which is relevant, not of methods of verification at all.

An example might make this clearer. How do I know the meaning of 'He is in pain'? Well, from my own case I know the meaning of 'I am in pain'. By decomposition (structural understanding of the latter assertion and how its components function together to make up a meaningful whole) I know what 'is in pain' means and by reassembly I know what 'He is in pain' means. Isn't that the analogist's argument? It is not that I imagine someone who, unlike me, can immediately experience everyone's pain, nor even that I grasp the meaning of 'He is in pain' by seeing the analogy between his directly 
experiencing his pains and me mine, and then relying upon the association of meaning with method of verification to think I now grasp the meaning of 'He is in pain'. No. It is that the meaning of 'He is in pain' is given by its truth-conditions, and these I understand by their analogy with the (directly epistemically accessible) truth-conditions of ' $\mathrm{I}$ am in pain'. It is something like a state-of-affairs which I am supposed to 'grasp' on the analogy with a directly experiencible state-of-affairs that is at the heart of the analogist's claim seen from this point of view. Of course this is not a theory of meaning and it isn't clear that there is any coherent theory which can 'back up' this initial 'model' or 'picture'. We will return to this problem shortly.

I have no intention here, of course, of even making reasonably coherent suggestions about just what a theory of meaning would be which would allow for the embedding in it of a theory of legitimate projection of meaning by means of analogy. Rather, I will just note four aspects of the problem of meaning by analogy which are of some importance, aspects which will come under discussion, I think, no matter what the general meaning theory is to be which allows for analogical projection of the comprehension of the meaning of assertions.

\section{A. Skepticism}

If we agree that we can grasp the meaning of proposition on the basis of their analogy with previously understood propositions, we must realize that we have opened ourselves up to the possibility of meaningful assertions relative to which no grounds of warranted assertability and deniability are forthcoming (or, perhaps, even could be forthcoming). This is not to say that the believer in semantic analogy must be committed to the comprehendability of at least some 'undecidable' propositions, but only that he might be.

In this way he surely differs from the verificationist. If the very meaning of the proposition is fixed by the warranting conditions for it, one need never fear that one will come upon a meaningful assertion for which warranting conditions are not forthcoming. Probably, also, in this the believer in semantic analogy differs from the holist as well, although it isn't as clear in this 
case as it is in the case of the verificationist account of meaning. For the holist the meaning of the assertions not asserting the existence of conditions directly open to epistemic access is fixed by the role the assertion plays in a total theory. But the total theory must be responsible, presumably only to the empirical facts which could serve to confirm or disconfirm it. Now if we take it as a sufficient condition of theoretical synonymy (two theories being equivalent in the sense of 'saying the same thing') that the two theories have a common body of observational consequences, then once again skepticism has been undercut. We need not fear the existence of alternative theories which cannot be judged relative to one another because they have their empirical consequences in common, for in the case of common consequences we just assimilate the theories together by characterizing them as synonymous. And of course the holist will not countenance our asking the question of how we test individually the assertions of the theory for acceptability, since it is just this consideration of the assertions 'one at a time' which he will not allow.

But if it is true that both verificationism and holism amount to a denial of realism for the assertions whose truth-conditions lie outside the range of direct epistemic accessibility, then it is hardly a surprise that the semantic analogist has greater concern than they over the problems of skepticism. For he takes these 'theoretical' assertions to be true and false, as are the 'observational', and their components to have genuine reference, extension, etc. And yet he freely admits that the truth-conditions for the assertion are not the sort we can simply observe to either be or not be the case. If I take it that 'He is in pain' is an assertion of the existence of a mental state of another, not open to my epistemic access, then of course I will worry about how I can ever really know whether or not someone is in pain in a manner which will not bother at all someone to whom a nothing would do as well as a something as far as the meaning of 'pain' was concerned.

\section{B. The Limits of Meaning by Analogy}

To just what extend can we project meaning out of our basis sentences and on to new assertions by means of semantic analogy? Perhaps, if we take basic sentences as reporting the contents of immediate subjective awareness, we could project meaning onto assertions about the external world. At least Locke thought that we could although Berkeley's familiar criticisms make 
us pause even here. Perhaps, using a projection of meaning by analogy, we can understand sentences attributing mental states to others on the basis of our understanding of mentalistic assertions about our own private mental states. Although, again, Wittgenstein would have us doubt even this. But to what extent can we support, for example, a realistic interpretation of theoretical assertions in science by a program which initially attributes intelligible meaning to these assertions on the basis of their semantic analogy with assertions of the observation language assumed to be understood?

Perhaps it isn't too unreasonable to view bacteria as, among other things, tiny objects too small to see. Even for molecules the analogy is 'good enough' to allow us to at least plausibly maintain that we understand the kinetic theory of gases on the analogy with a box filled with rapidly moving billiard balls. But when we get to electrons, quarks, photons, or worse yet, virtual intermediate massless bosons, charm, etc. what earthly use can we make of analogy as a source of meaning?

I think there are two things that can be said. First, even in some pretty recherche cases there is still a certain amount of theoretical predication going on which can at least plausibly be argued to be understood in the analogical way. Even if virtual particles are sufficiently remote from dust particles in their properties that to speak of them as particles at all is just to mislead, still they do have, perhaps, spatiotemporal location, momentum, energy, etc. and perhaps these can be understood on analogy with those features when predicated of objects of experience. Second, insofar as the features of things cannot be understood on any analogy whatever with features of the elements of experience, we can, at this point, always retreat to an instrumentalistic denial of full meaning to the predicates altogether.

Electric charge is just the disposition to behave in certain ways under certain test conditions; the psi-function of quantum mechanics is just an 'intervening variable' connecting test conditions and results of measurements, both characterized in the old observational vocabulary, but having no independent meaning or reference of its own, etc. Witness Bohr's insistence that our body of real concepts will always be restricted to those familiar to us from everyday experience, the rest of the theoretical apparatus to be understood only functionally relative to assertions framed in this original vocabulary.

Now it may very well be that the disposition on our part to hold to a realistic interpretation of theoretical assertions when they are framed in the 
familiar vocabulary of observational experience, and retreat to instrumentalism only when terms are invoked which can't be understood analogically on the basis of observational predicates is a more psychological prejudice on our part. Certainly this would be the claim of the orthodox verificationist and the holist. But $I$ think it would be as premature to dogmatically accept such a claim and reject our 'intuitions', vague as they may be, as it would be to rashly accept the Humean claim that our belief in the uniformity of nature is but a reflection of psychologically explicable prejudice and not a rational and rationalizable approach to an attempt to know what goes on in the world.

\section{Legitimate vs Illegitimate Analogy}

As noted earlier a primary assault on the very notion of semantic analogy proceeds by attempting to show that the principles the analogist uses to allow the projection of meaning from sentence to sentence - basically, I have claimed, the principle of decomposition and reassembly - are such that they lead to the claimed intelligibility a prima facie meaningless utterances ('It is five o'clock on the sun', 'The number three is thinking of Vienna'. etc.). Now obviously the analogist can reply that he doesn't accept any decomposition and reassembly of sentential components as guaranteeing a transfer of meaningfulness from the original sentences decomposed to the newly reassembled utterances. There must be some restrictions on the range of decomposition and reassembly.

But how these restrictions are to be formulated is going to be a matter of some difficulty for the analogist. I do not believe that any naive attempt in terms, say, of a categorization of vocabulary into semantic classes and an attempt to delimit legitimate rules of assembly in terms of these classes will do the job. Even the most obvious rules we think of will meet a verificationist challenge, we might note. Mere substitution of indexicals is itself dubious, according to the verificationist, for, according to him, I don't understand 'He is in pain' on the basis of 'I am in pain' even though both indexicals refer to persons. Again, if some interpreters of relativity are right, Einstein showed that we were mistaken in thinking that we could understand the simultaneity relation when predicated of events at a distance from one another, just because we understood it when predicated of spatially coincident events. Now the verificationist might be wrong about these cases, but how is the analogist to convince him without a fullfledged theory of the legitimacy of 
analogical projection of meaning and without an analysis of the limits of legitimate meaning projection by analogy?

The comparison with the verificationist program is interesting here. The verificationists provided both a criterion of meaningfulness, and, much more interesting, a doctrine about meaning. The two fit together in an obvious way: to specify meaning is to give the conditions of warranted assertability and deniability. In the absence of such conditions, the assertion is meaningless. What is the analogist's theory of meaning to be, and how, relative to it, are the limits of legitimate projection of meaning by analogy to be characterized?

\section{A Theory of Meaning}

We now come to the most fundamental problem with the doctrine of the projectability of meaning by analogy. Into just what theory of meaning and the knowability of meaning is it to be inserted as a viable part?

The theory is one which takes as fundamental in meaning theory truthconditions and our 'grasp' of them. To understand the meaning of a proposition is to know what it asserts, i.e. to understand what it is for it to be true and what it is for it to be false. We can 'grasp' a truth-condition by having it directly accessible to us (we are immediately aware of the conditions asserted to be the case for the 'observation sentences') or we can 'grasp' a truthcondition by seeing its analogy to a truth-condition already comprehended by us, that is by 'constructing' the new truth-condition out of components available to us from their role in antecedently comprehended truth-conditions according to structural principles of constituting truth-conditions out of objects and properties also familiar to us from understood cases.

But what all this means is far, far from clear. It smacks all too much of a 'picture in the mind' theory of meaning, where comprehension of assertions is viewed in the manner of an association of sentences with pictures and of novel sentences with new pictures made up out of pieces cut from old ones and glued together in novel ways. This won't do, of course, as a theory of meaning. But just what does the analogist have to put in its place? In particular what does he have which is informative over and above the trivialities which tell us that to understand the meaning of a sentence is to grasp its truth-condition which is, after all, just to know what the sentence means? One thing 'grasping a truth-condition' cannot mean, of course, is knowing how to determine the truth or falsity of the proposition. For, according to this view and in direct conflict with verificationism in any of its forms, 
we can perfectly well know the meaning of a proposition and yet have no idea whatever how one could possibly determine whether it be true or false. And it can't mean simply knowing the necessary and sufficient conditions for the truth of the proposition.

Despite the difficulties such a theory of meaning and the comprehension of meaning obviously would meet, it still might be worth some effort to find such a theory. To convince you of that let me end with a (familiar) little parable. Exploring a distant planet we come upon some entities. Utterly unlike any living creatures we are familiar with we still find their behavior complex enough to offer an account of it in terms of posited internal functional states. Let us call one such state being in 'grinch'. Our complex functionalist theory sometimes leads us to say 'That one is in the grinch state.' After a while a particularly imaginative alien-anthropologist suddenly sees close formal parallels between the functional organization of the internal states of the creatures and the 'program' of functional organization of human mental states. In this formal parallel, 'being in the grinch state' is located just about where 'being in pain' is located in our description of the mental life of men. So, speculating, he says: Perhaps being in grinch is being in pain'.

Now we are not concerned with whether or not he is right. Or even if he ever has any good reason for believing he is right in identifying grinchness with pain. The point is, do we not believe that in coming to entertain the proposition 'That thing is in pain' he has come to entertain a novel proposition different from merely believing 'That creature is in the grinch state', and, indeed, a proposition he can understand only because he knows what pain is (presumably, but not necessarily, from his own case). But his means for establishing whether or not a creature is in pain are, of course, the very conditions he uses for establishing whether or not a creatures grinches. Yet the proposition he now takes warranted by 'grinching behavior' is not merely that the creature is in a grinch state, but that it is in pain. If you accept that a novel proposition has been entertained by him, then you accept the claim that there is more to meaning of an assertion than its conditions of warranted assertability and deniability. And if you accept the claim that this additional element is a grasp of meaning which comes about from understanding the meanings of the components in the novel assertion from understanding the role they had in previously understood assertions, then you ought to pursue an analogical theory of meaning projection, elusive as such a theory might be. 


\section{NOTES}

* Work on this paper was partially supported by a research grant of the National Science Foundation. Grant No. SOC 76-22334.

1 M. Dummett, 'What is a theory of meaning? (II)', in G. Evans and J. McDowell (eds.), Truth and Meaning (Oxford, 1976), pp. 67-137. The argument discussed above is on pp. 98-101. 